\title{
Optical rhinometry in nasal provocation testing
}

\author{
Edyta Krzych-Fałta ${ }^{1}$, Adam Sybilski ${ }^{1,2}$, Oksana Wojas ${ }^{1}$, Bolesław Samoliński ${ }^{1}$
}

${ }^{1}$ Department of Environmental Risk Prevention and Allergology, Faculty of Health Sciences, Medical University of Warsaw, Warsaw, Poland Head of the Department: Prof. Bolesław Samoliński MD, PhD

2Department of Pediatrics and Neonatology, Central Clinical Hospital of the Ministry of Interior, Warsaw, Poland

Head of the Department: Adam Sybilski MD, PhD

Postep Derm Alergol 2015; XXXII (6): 449-454

DOI: $10.5114 /$ pdia.2015.56099

\begin{abstract}
Introduction: Optical rhinometry (ORM) is a technique that allows for direct continuous measurement of changes in blood flow (optical density) in nasal vessels, at the same time analyzing changes per unit of time in the evaluated variable: intravascular blood flow and oxygen saturation.

Aim: To assess the extent to which ORM can be used as an objective measure of nasal mucosal edema following a nasal allergen provocation test in a group of patients with allergic rhinitis versus healthy controls.

Material and methods: The study group included 60 subjects: 30 patients diagnosed with an allergy to common environmental allergens (dust mites/grasses) and 30 healthy controls. The method used in the study was a nasal provocation test with an allergen, with a standard dose of a control solution and an allergen (5,000 SBU/ml) administered using a calibrated atomizer into both nostrils in room temperature.

Results: The mean delay to the onset of nasal mucosal response as measured by ORM was 3.15 min and the level of light extinction returned to baseline after $28.15 \mathrm{~min}$ (change in optical density 0.431). These objective changes in optical density strongly correlated with subjective perception measured via a visual analog scale. Conclusions: Optical rhinometry is a valuable tool for nasal allergen provocation testing.
\end{abstract}

Key words: nasal allergen provocation test, optical rhinometry, optical density, onset/maximum nasal mucosal edema, visual analogy scale.

\section{Introduction}

Optical rhinometry (ORM) is a technique that allows for direct continuous measurement of changes in blood flow (OD - optical density) in nasal vessels, at the same time analyzing changes per unit of time in the evaluated variable: intravascular blood flow and oxygen saturation [1, 2] (Figure 1). Rhinometry is performed with an emitter and detector placed on opposite sides of the bridge of the nose. The device emits pulses of infrared light with a mean wavelength of 600-800 $\mu \mathrm{m}$ through nasal tissues, which are detected with a frequency of $0.2 \mathrm{~s}$. Unlike rhinomanometry, ORM offers real-time measurements, irrespective of subject compliance, in the case of polyps, perforation, and deviated septum, as well as technical ease of measurement $[3,4]$. Popularized mainly by German researchers (Wüstenberg EG, Hampel U, Schleicher E, Hüttenbrink KB, Zahnert T), this method has been gaining more and more approval in recent years, especially in assessing the early phase of an allergic reaction via nasal allergen provocation testing (NAPT), which plays an increasing role in allergic rhinitis diagnostics due to its high sensitivity and specificity. Although NAPT simulates the natural exposure to an allergen only to a certain extent (single allergen dose), it is still a valuable source of information on the patients' condition. In the case of significant inconsistencies in the patients' history, skin prick tests or serum IgE tests are often the basis for patient qualification for immunotherapy $[5,6]$.

\section{Aim}

The purpose of this study was to assess the applicability of ORM as an objective technique to verify the degree of nasal mucosal edema following nasal allergen administration in patients with allergic rhinitis (AR) versus the healthy controls ( $\mathrm{HC})$.

Address for correspondence: Edyta Krzych-Fałta PhD, Department of Environmental Risk Prevention and Allergology, Faculty of Health Science, Medical University of Warsaw, 1a Banacha St, 02-097 Warsaw, Poland, phone: +48 225992039 , fax: +48 225992042 , e-mail: e.krzych@gmail.com Received: 27.02.2015, accepted: 14.06.2015. 

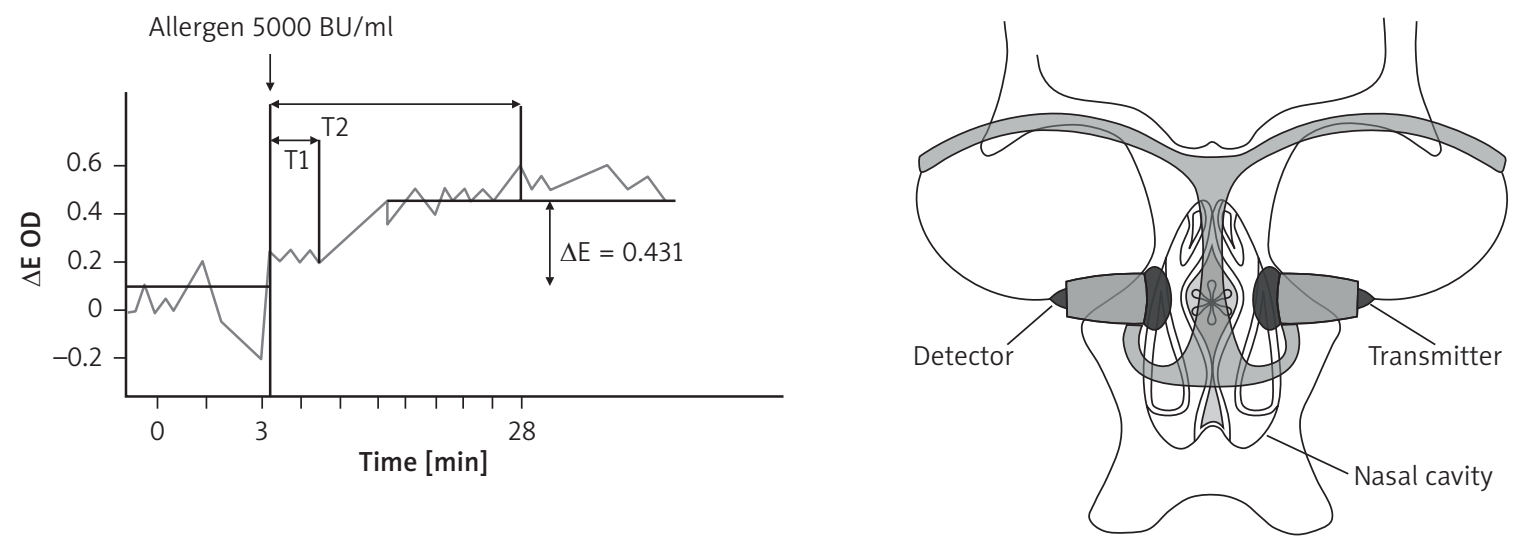

\begin{tabular}{lccc}
\hline Evaluated parameter & AR & HC & Value of $p$ \\
\hline OD & 0.431 & 0.010 & $<0.05$ \\
\hline T1/T2 & $3.15 / 28.15$ & $0.800 / 0.930$ & $<0.05$ \\
\hline
\end{tabular}

$O D$ - optical density, T1/T2 - onset/maximum nasal mucosal edema, AR - allergic rhinitis, HC - healthy controls.

Figure 1. The onset and maximum optical density during optical rhinometry

\section{Material and methods}

Nasal allergen provocation testing performed by qualified medical personnel in an outpatient setting is the simplest and most accurate model of natural exposure of the nasal mucosa to an allergen, measured in time. According to the Report by the Committee for Upper Airways Allergies, NAPT may be defined as a method "restoring the response of the upper airways to natural exposure to allergens or irritants and doing research into the pathophysiology of the upper airways by testing potential biochemical mediators" $[6,7]$. The nasal mucosal reaction to the administered allergen is instantaneous and involves mainly activation of cells (mast cells, macrophages); activated cells are coated with antibodies, which results in the release of tryptase, histamine, CysLT (custeinyl leukotriens), and $\mathrm{PGD}_{2}$ (prostaglandin 2) [6, 7]. These mediators, in turn, cause local stimulation of sensory nerve receptors and blood vessels within the nasal mucosa. Moreover, during the early phase of inflammation, mast cells release chemotactic agents and platelet-activating factor, which contribute to the development of inflammation $[6,8]$. The immediate allergic reaction typically lasts approximately 20-30 min and may continue into the late phase, which begins approximately $4 \mathrm{~h}$ after nasal allergen administration [6, 9]. The International Committee on Objective Assessment of the Nasal Airways and the Polish 2010 Nasal Provocation Test Standardization Consensus recommend that NAPT be assessed concomitantly via an objective and subjective technique per unit of time [6, 7]: however, fail to indicate how to interpret test results.

\section{Study group}

The study group comprised 60 subjects: 30 patients diagnosed with allergic rhinitis (AR) to common environmental allergens: dust mites (Dermatophagoides pteronyssinus) and grasses, and $30 \mathrm{HC}$ with no symptoms of allergy (Table 1). In order to increase effectiveness of NAPT, the study population was selected based on the dominant allergen (dust mites or grass pollens) identified via skin prick tests. The study group of

Table 1 . Characteristics of the 30 study participants

\begin{tabular}{lcc}
\hline Parameter & $\begin{array}{c}30 \text { patients with AR } \\
(14 \text { women, 16 men })\end{array}$ & $\begin{array}{c}30 \text { healthy controls } \\
(13 \text { women, 17 men })\end{array}$ \\
\hline Age [years] & $27.33 \pm 5.665$ & $30.63 \pm 6.037$ \\
\hline Height $[\mathrm{cm}]$ & $172.90 \pm 8.767$ & $176.03 \pm 8.588$ \\
\hline Weight $[\mathrm{kg}]$ & $72.83 \pm 13.570$ & $74.10 \pm 13.583$ \\
\hline Positive results of skin prick tests & $\begin{array}{c}\text { SAR: } 11.0 \pm 3.326 \mathrm{~mm} \text { vs. } \\
\text { histamine } 3.89 \pm 0.832 \mathrm{~mm} \\
\text { pAR: } 8.92 \pm 3.315 \mathrm{~mm} \text { vs. } \\
\text { histamine } 3.42 \pm 0.669 \mathrm{~mm}\end{array}$ & \\
\hline
\end{tabular}

$S A R$ - Seasonal allergic rhinitis, $p A R$ - perennial allergic rhinitis. 
30 RA patients was not stratified into seasonal and chronic allergy due to the small sample size.

Subjects were qualified for the study based on:

- documented history of allergy to a given allergen (at least 3 years of symptoms, without medical treatment or specific immunotherapy),

- a positive skin prick test,

- computed tomography scans of paranasal sinuses that excluded inflammation,

- preserved patency of the osseous part of nasal passages.

Exclusion criteria:

- at least 6 weeks after a pollination period (airborne allergens) preventing "sensitization" prior to NAPT,

- nasal malformations, choanal atresia, nasal septum perforation, significant nasal septal deviation,

- nasal polyps,

- atrophic rhinitis,

- less than 6 weeks from the end of symptomatic allergic rhinitis; patients with seasonal rhinitis were evaluated in the period from March to May,

- any vaccinations (according to the vaccination schedule, with immunizing agents) administered within one week prior to the study,

A

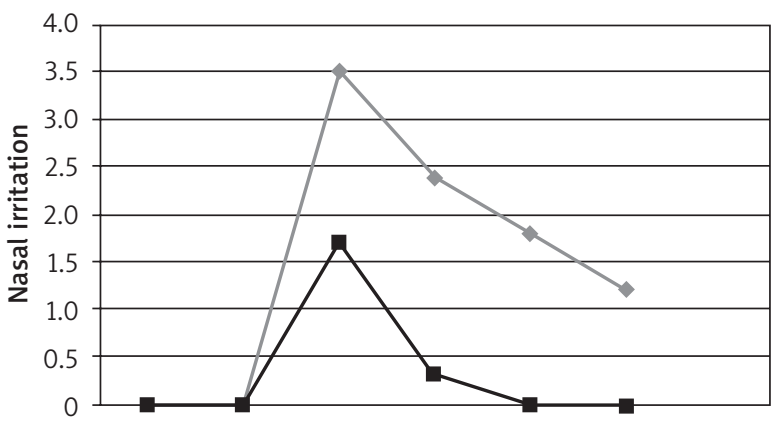

$1^{\text {st }}$ examination Control $5 \mathrm{~min} 10 \mathrm{~min} 15 \mathrm{~min} 20 \mathrm{~min}$ $\rightarrow \mathrm{AR} \rightarrow \mathrm{HC}$

C

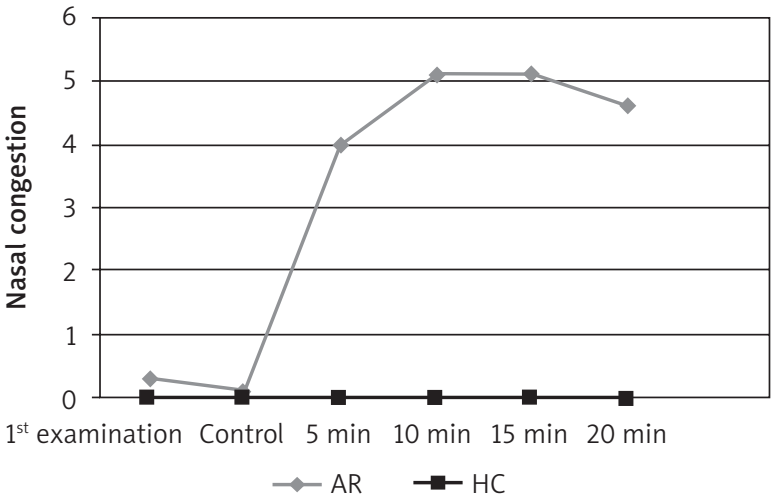

- less than 8 weeks after nasal surgery (particularly any inferior turbinate reduction procedures),

- acute upper respiratory tract infections within 2-4 weeks prior to the study,

- sinusitis, including frontal sinusitis,

- bronchial asthma,

- hypertension and other cardiovascular conditions,

- pregnancy and lactation,

- active or passive smoking.

\section{Method}

The method used in the study was NAPT with the use of 5,000 standardized biological units (SBU)/ml (Allergopharma) administered with a calibrated atomizer into both nostrils at room temperature at a dose of $0.2 \mathrm{ml}$. The nasal reaction was assessed via light extinction curve analysis of infrared light emitted and detected by an optical rhinometer as well as via a visual analog scale (VAS) (nasal irritation, sneezing, congestion, rhinorrhea) at 5, 10, 15, and 20 min after allergen provocation. The study was conducted owing to a grant from the Pomeranian Ministry of Science and Higher Education (N N402 520839) and was approved by the

B

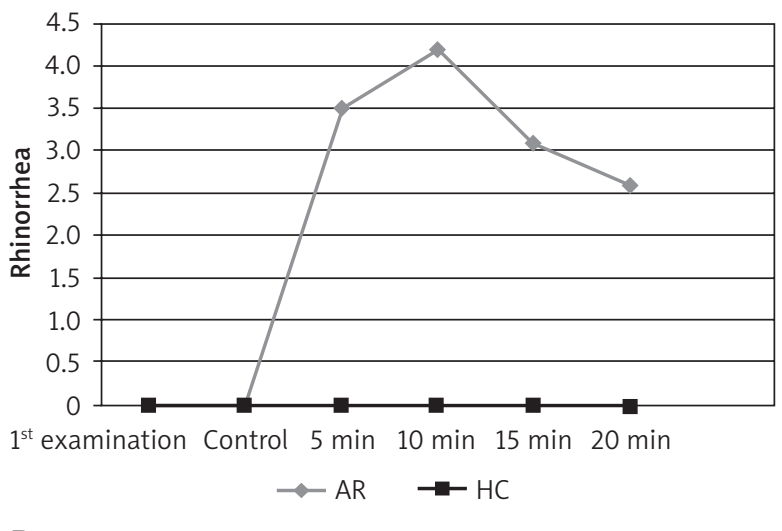

D

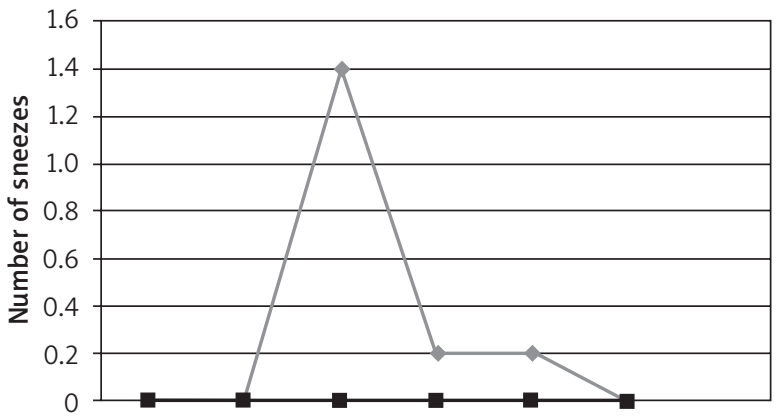

$1^{\text {st }}$ examination Control $5 \mathrm{~min} 10 \mathrm{~min} 15 \mathrm{~min} 20 \mathrm{~min}$

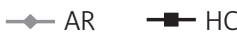

AR - Allergic rhinitis, $\mathrm{HC}$ - healthy controls, ${ }^{*} p<0.05$.

Figure 2. Curves showing mean nasal symptoms (VAS) during NAPT 
Institutional Review Board at the Medical University of Warsaw (KB/79/2009).

\section{Statistical analysis}

Student's t-test and Pearson's correlation coefficient were calculated in order to determine the relationship between variables and the strength of the relationship. The Levene's test was used to estimate the uniformity of means. Value of $p<0.05$ was considered to be statistically significant.

\section{Results}

We observed significant differences between study groups in terms of nasal symptoms such as nasal irritation, rhinorrhea, nasal congestion, number of sneezes within 5 to $20 \mathrm{~min}(p<0.05)$ (Figure 2). The greatest severity of nasal irritation and increased number of sneezes were observed in the AR group at $5 \mathrm{~min}(p<0.05)$, rhinorrhea at 10 min $(p<0.05)$, and nasal congestion at 10 , 15 , and $20 \mathrm{~min}(p<0.05)$ in comparison with the control group. The first nasal symptoms in the form of nasal irritation developed 5 min after allergen administration; its change in time showed significant differences from time 0 following allergen administration to $5 \mathrm{~min}(p=0.006)$, from 10 to $15 \mathrm{~min}(p=0.034)$, and from $20 \mathrm{~min}$ to $4 \mathrm{~h}$ $(p=0.018)$. The number of sneezes recorded after $5 \mathrm{~min}$ showed no significant change during the early phase of allergic reaction $(p=0.85)$.

A

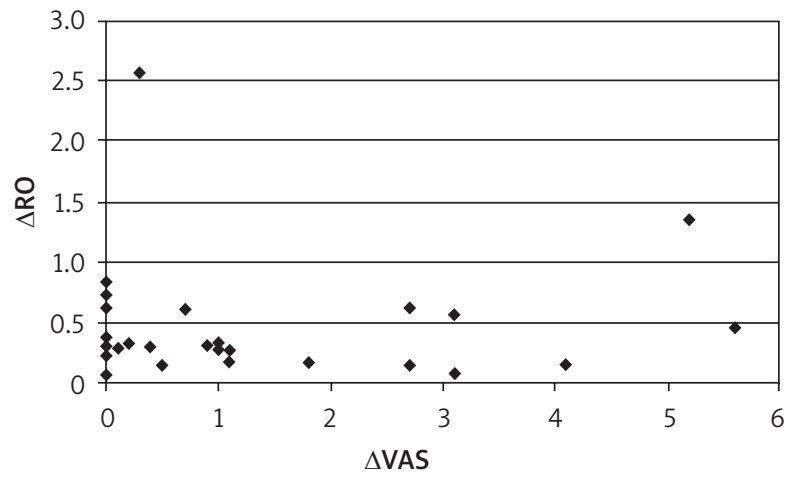

C

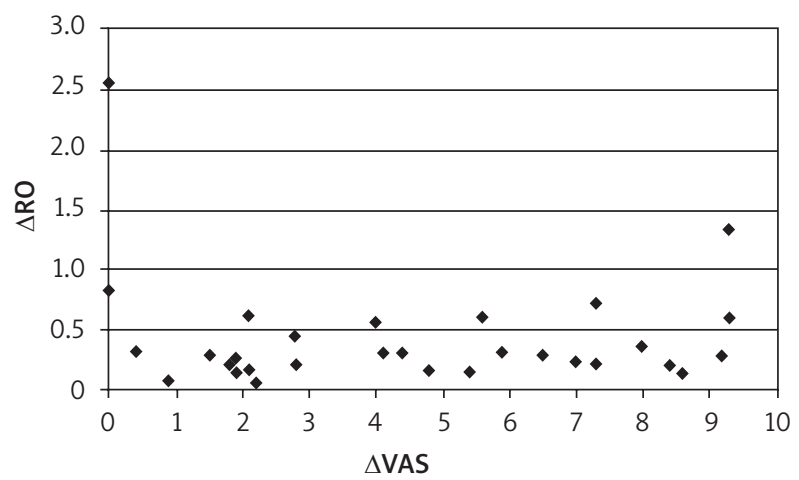

In the AR group, there were significant differences both in terms of mean change in infrared light absorption and in the onset of allergic reaction in comparison with the control group $(p<0.05)$ (Figure 1$)$. The mean onset of mucosal reaction on the optical rhinometry curve was recorded after $3.15 \mathrm{~min}$, while light extinction returned to its lowest (baseline) level after 28.15 min. The mean value of infrared light absorption in the AR group in comparison with that in the control group was OD $=0.431$ $(p<0.05)$. We observed strong correlations between symptoms recorded via the VAS (nasal irritation $r=0.13$, $p=0.016$; rhinorrhea $r=0.493, p<0.005$; nasal congestion $r=0.333, p=0.009$ ) and ORM between 5 and 20 min following nasal allergen administration (Figure 3).

\section{Discussion}

Optical rhinometry (method introduced for nasal cavity examination in Germany in 2004) [10] as an objective assessment technique, also used in NAPT due to its simplicity, has been a valuable source of diagnostic information in rhino-allergology. The observed objects along the course of the ORM curve are usually associated with sneezing, pronounced facial muscle movements, or shifts in body position [10]. Tillmann et al. demonstrated distinct variations in optical densities in a group of 52 healthy subjects aged $21-80$; in the $21-40$ age group OD increased by 0.14 , in the $41-60$ age group - by 0.22 , and in the 61-80 age group - by 0.31 [11]. The increase in OD

B

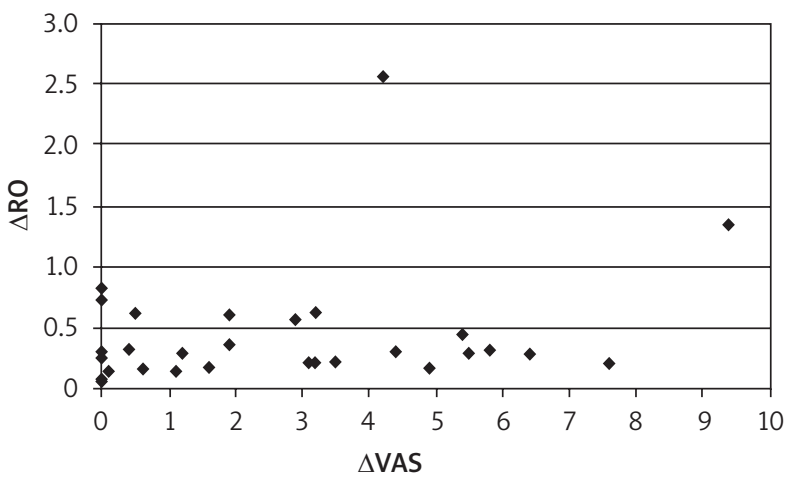

Figure 3. The relationship between symptoms recorded via VAS and nasal mucosal edema recorded via optical rhinometry. A - Nasal irritation $(r=0.13, p=0.016)$, B - rhinorrhea $(r=0.493, p<0.005), C-$ nasal congestion $(r=0.333$, $p=0.009)$ 
with age has been associated with increased vascular stiffness [12] and concomitant conditions attributed to old age. There have been few animal studies on this subject, which provides insufficient evidence as to the rationale for using ORM in nasal patency assessments [13]. The obtained results are in the form of absolute numbers, which cannot be compared between, or standardized for, individual study groups or subjects [14]. In comparison with other techniques, such as rhinomanometry, where NAPT is evaluated based on the increase in intranasal pressure and peak nasal inspiratory flow (PNIF) tests assessing the flow of a specific volume of air per unit of time (minute), ORM has a distinct advantage in terms of subject compliance. Moreover, ORM shows a strong correlation with active anterior rhinomanometry (Wüstenberg; $r=0.84$ ) [2], acoustic rhinometry (Cheung; minimum cross-sectional area (mCSA)- $1 r=-0.6, p=0.03$ and mCSA-2r $=-0.64, p=0.02$ ) [4], and subjective symptom assessment via a VAS during NAPT [14, 15].

Our study showed the ORM-measured onset of reaction at 3.15 min with simultaneous development of nasal irritation ( $p=0.042)$. At $10 \mathrm{~min}$, rhinorrhea occurred, with nasal congestion reported as the predominant symptom at that time point; also, the changes over time from allergen administration to 5 min $(p=0.01)$ were statistically significant. These ORM-recorded changes over time correlated with symptoms reported by subjects (nasal irritation $r=0.13, p=0.016$; rhinorrhea $r=0.493, p<0.005$; nasal congestion $r=0.333, p=0.009$ ). Our observations are consistent with those by Wüstenberg, who demonstrated the strongest correlation between ORM values and rhinorrhea $(r=-0.63)$, with a mean decrease in light extinction maintained at a level of OD 0.435 [2]. Cheung et al. also observed a positive correlation between the change in light extinction and the increase in symptoms as measured via VAS $(r=-0.60 ; p=0.03)$ [4]. Similar correlations were reported by Luong et al., who demonstrated a strong correlation between VAS symptoms and ORM results $(r=0.79, p=0.00003)$ [16]. Wüstenberg et al. reported a slightly longer delay, of $7.46 \mathrm{~min}$, to optical density reduction $(O D=0.410)$, which strongly correlated with rhinomanometric parameters [12]. Additional tests in children and adults showed variations in the onset and duration of maximum nasal mucosal edema, with onset at $120 \mathrm{~s}$ and maximum edema at $330 \mathrm{~s}$ in children and onset at $156 \mathrm{~s}$ and maximum edema (complete light extinction) at $466 \mathrm{~s}$ in adults; these differences were not statistically significant $[2,9,10]$. Subjects reacted to provocation with histamine noticeably more rapidly than to provocation with an allergen [17]. Ohm and Juto observed a more pronounced reaction to histamine administration in the group of healthy subjects versus the group of subjects with hyperactive nasal mucosa [18]. Conversely, Mittenzwey et al. in a small group of children reported a significant change over time of $O D=0.37$ during NAPT and a slightly lower OD of 0.32 with histamine provocation after 2.39 min [14]. This distinct variability was likely due to the varied size of intranasal structures and mucosal thickness between study groups [17]. Some study results confirm the utility of ORM in allergic rhinitis diagnostics as evidenced by strong correlations [19, 20] whereas other study results clearly call the usefulness of this technique into question [21-23].

\section{Acknowledgments}

This study was supported by the Pomeranian Ministry of Science and Higher Education (N N402 520839).

\section{Conflict of interest}

The authors declare no conflict of interest.

\section{References}

1. Wüstenberg EG, Hüttenbrink KB, Hauswald B, et al. Die optische Rhinometrie. Kontinuierliche, direkte Messung der Schwellung der Nasenschleimhaut be Allergenprovokation. HNO 2004; 52: 798-806.

2. Wüstenberg EG, Zahnert T, Hüttenbrink KB, et al. Comparison of optical rhinometry and active anterior rhinomanometry using nasal provocation testing. Arch Otolaryngol Head Neck Surg 2007; 133: 344-9.

3. Mittenzwey H, Wüstenberg EG, Leupold KW. Optical rhinometry: application on children and adolescents for nasal provocation tests. Pediatr Allergy Immunol 2007; 18: 372-7.

4. Cheung EJ, Citardi MJ, Fakhri S, et al. Comparison of optical rhinometry to acoustic rhinometry using nasal provocation testing with Dermatophagoides farinae. Otolaryngol Head Neck Surg 2010; 143: 290-3.

5. Bousquet J, Khalataev N, Alvaro A, et al. Allergic rhinitis and its impact on asthma (ARIA) 2008. Alergia Astma Immunol Klin 2008; 13 (Suppl. 1): 3-39.

6. Samoliński B, Krzych-Fałta E. Definition of nasal provocation test and nasal allergen challenge, classification. Postep Derm Alergol 2010; 27: 166-9.

7. International Consensus Report on Diagnosis and Management of Rhinitis. International Rhinitis Management Working Group. Allergy 1994; 49 (19 Suppl.): 1-34.

8. Samoliński B, Rapiejko P. The early reaction after nasal airway challenge with alergen. Postep Derm Alergol 2010; 27: 170-2.

9. Samoliński B, Rapiejko P. The late-phase reaction after nasal airway challenge with alergen. Postep Derm Alergol 2010; 27: 173-6.

10. Wüstenberg EG, Zahnert T. Dokumentation von endonasalen Blutvolumenänderungen mittels optischer Rhinometrie. HNO 2006; 54: 99-104.

11. Tillmann HC, Laske A, Bernasconi C, et al. Age determines vascular reactivity as measured by optical rhinometry. Eur J Clin Invest 2009; 39: 1010-6.

12. Abhayaratna WP, Barnes ME, O'Rourke MF, et al. Relation of arterial stiffness to left ventricular diastolic function and cardiovascular risk prediction in patients $>$ or $=65$ years of age. Am J Cardiol 2006; 98: 1387-92.

13. King D, Etzel JP, Chopra S, et al. Human response to alpha2adrenergic agonist stimulation studied in an isolated vascu- 
lar bed in vivo: biphasic influence of dose, age, gender, and receptor genotype. Clin Pharmacol Ther 2005; 77: 388-403.

14. Mittenzwey H, Wustenberg EG, Leupold W. Optical rhinometry: application on children and adolescents for nasal provocation tests. Pediatr Allergy Immunol 2007; 18: 372-7.

15. Hampel U, Schleicher E, Wustenberg EG, et al. Optical measurement of nasal swellings. IEEE Trans Biomed Eng 2004; 51: 1673-9.

16. Luong A, Cheung EJ, Citardi MJ, et al. Evaluation of optical rhinometry for nasal provocation testing in allergic and nonallergic subjects. Otolaryngol Head Neck Surg 2010; 143: 284-9.

17. Wüstenberg EG, Huttenbrink KB, Hauswald B, et al. Optical rhinometry. Continuous, direct measurement of swelling of the nasal mucosa with allergen provocation. Real-time monitoring of the nasal provocation test using optical rhinometry. HNO 2004; 52: 798-806.

18. Ohm M, Juto JE. Nasal hyperreactivity. A histamine provocation model. Rhinology 1993; 31: 53-5.

19. Naito K, Miyata S, Saito S, et al. Comparison of perceptional nasal obstruction with rhinomanometric and acoustic rhinometric assessment. Eur Arch Otorhinolaryngol 2001; 258: 505-8.

20. Simola M, Malmberg H. Sensation of nasal airflow compared with nasal airway resistance in patients with rhinitis. Clin Otolaryngol Allied Sci 1997; 22: 260-2.

21. Kim CS, Moon BK, Jung DH, et al. Correlation between nasal obstruction symptoms and objective parameters of acoustic rhinometry and rhinomanometry. Auris Nasus Larynx 1998; 25: 45-8.

22. Hirschberg A, Rezek O. Correlation between objective and subjective assessments of nasal patency. ORL J Otorhinolaryngol Relat Spec 1998; 60: 206-11.

23. Numminen J, Ahtinen M, Huhtala H, Rautiainen M. Comparison of rhinometric measurements methods in intranasal pathology. Rhinology 2003; 41: 65-8. 